\title{
RECENT STUDIES ON COUPLING BETWEEN TRANSLATIONAL TRANSPORT AND REACTIVITY AT MOLECULAR SCALE: MODELS AND EXPERIMENTATION
}

\author{
J. C. ANDRE, F. BAROS, A. BOUCHY, M. BOUCHY, W. DONG, \\ J. DUHAMEL, A. T. REIS E. SOUSA $\uparrow$ and M. L. VIRIOT \\ GRAPP-URA 328 CNRS ENSIC-INPL, BP 451, 1, rue Grandville, \\ F 54001 NANCY Cedex (France)
}

(Received November 1, 1989; in final form April 30, 1990)

Different theoretical models are presented concerning diffusion-influenced reactions. Pulsed laser systems allow time resolved fluorescence measurements of these kinetic phenomena. The connection between theory and experimentation is illustrated.

KEY WORDS: Laser applications, diffusion, chemical rates of reactions.

$\beta: \quad$ resonance integral

$D: \quad$ mutual diffusion coefficient

$\delta_{q q},:$ interaction integral

$\varepsilon: \quad$ dielectric constant

$E_{\text {int }}:$ ground-state/ground-state interaction energy

$E_{\text {int }}{ }^{*}$ : interaction energy between an excited molecule and a ground-state molecule

$\Phi: \quad$ configurational distribution function

$\phi: \quad$ repartition of $\mathrm{B}$ particles around $\mathrm{A}$

$\Phi_{q}: \quad$ atomic orbital

$g(r)$ : radial distribution function

$k(r)$ : rate of reaction

$k_{a}$ : apparent rate constant

$k_{B}$ : Boltzmann constant

$k_{c}$ : chemical rate of a bimolecular process

$K_{S V}:$ Stern Volmer constant

$k_{\infty}$ : rate constant at large times

$N$ : Avogadro number

$p_{A}(t)$ : the probability of existence of a molecule $\mathrm{A}$, in an infinite environment of B $\rho B: \quad B$ density in the medium

$\sigma$ : $\quad$ hard spheres diameter

† Centro Quimica Fisica Molecular, INIC-IST, Avenida Rovisco Païs, 1096 LISBOA Codex (Portugal). 
$\sigma^{\prime}: \quad$ reaction distance

$S_{q q}{ }^{\prime}:$ overlap of $p$ orbitals of the pair of atoms $q$ and $q^{\prime}$

$T: \quad$ absolute temperature

$U_{\text {inter: }}$ total interaction energy

$W: \quad$ well depth of a Lennard-Jones intermolecular potential

$X(r)$ : intermoleculart potential

$\Psi_{j}$ : molecular orbital

\section{INTRODUCTION}

Consider a pseudo two-dimensional fluid of hard spheres subject to Brownian motion, a probability of collision between two molecules, labelled A and B, can be defined in a closed medium at molecular scale. This probability depends on the positions of these two particles, and on their initial velocities. To define the time evolution of such dynamic systems, assuming no reaction, the Rice and Allnatt ${ }^{1}$ equation is used.

If the velocity and position are not correlated, on assumption justified at large time, the Smoluchowski ${ }^{2}$ equation must be solved.

The initial configurational distribution $\Phi$ is determined using the superposition principle, illustrated on Figure 1.

At first, the problem is simplified by choosing a reactional space or a predominant coordinate, usually the intermolecular distance. Starting, at time $t$, with a distribution $\phi$ of B particles around A, $\Phi$ is obtained by averaging the configurations. If a reaction does not occur, $\Phi$ must be stationary. Another method to obtain $\Phi$ is proposed by considering the configurations at successive times $t$ and $t+\tau$ so that $\langle\phi(t) \phi(t+\tau)\rangle \cong 0$ and by calculating $\Phi$ with

$$
\Phi=\frac{1}{N+1} \sum_{n=0}^{N} \phi(t+n \tau) \quad N \longrightarrow \infty
$$

These considerations lead theoretically to a statistical determination of the distribution of B around A.

In the case of a diffusion-influenced bimolecular reaction, the use of the superposition principle leads to the modified Smoluchowski equation:

$$
\frac{\partial \Phi}{\partial t}=\operatorname{div}\left(D \operatorname{grad} \Phi-X \frac{D}{k_{B} T} \Phi\right)-k(r) \Phi
$$

where $k(r)\left(s^{-1}\right)$ is the rate of reaction, between A and B located at a distance $r$.

This equation is generally used with some refinements to treat the molecular coupling between transport and reactivity. However one must know the validity of this expression for which the molecular velocity is not taken into account; it is of prime importance to estimate the parts of the initial configuration and of the reactional mechanism on the apparent kinetics. 
(1)
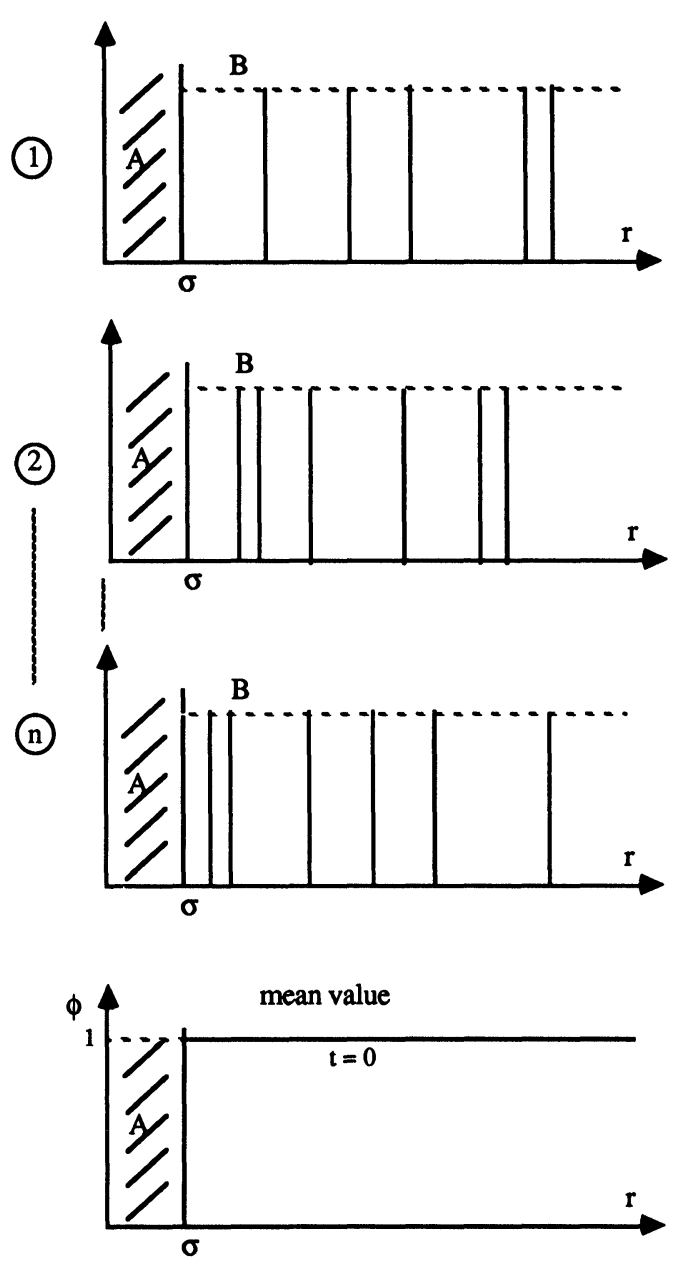

Figure 1 Use of the superposition principle in the case of random distribution of molecules B around molecules A.

The aim here is to point out how these parameters occur, and, in a second part, how the experiments can be explained by theoretical kinetic models.

The electronic excitation can lead to very reactive species and it is possible to observe the coupling between transport and reactivity by selection of $\mathrm{B}$ and by accommodating the medium where the reaction takes place. Moreover, if $\mathrm{A}$ is fluorescent, its evolution can be scanned by photophysical methods, time resolved or not. For time resolved experiments, the picosecond laser techniques allow a good kinetic description of the phenomena.

Next, the use of laser photophysics techniques in this area is discussed. 


\section{KINETIC MODELS}

As previously mentioned, the initial point is to know if the Smoluchowski equation can be used for the treatment of kinetic experiments when there is a strong coupling between molecular transport and reactivity. For this purpose, a model has been developed, based on a technique of molecular dynamic which allows a better understanding of the limits of validity of this equation. Starting from this new concept, it is possible to verify the kinetic models in some particular cases. Some significant examples are presented on Table 1, where the space affected by the reaction is assumed to have a spherical symmetry and an infinite dimension.

A similar treatment can be applied for the other cases (see references at the end).

Table 1 Some examples of photophysical systems discussed in this paper

\begin{tabular}{cl}
\hline $\mathrm{A}+\mathrm{B} \rightarrow \ldots \ldots$ & Diffusion limited reaction \\
$\mathrm{A}+\mathrm{B} \rightarrow \ldots \ldots$ & Coupling between transport and reactivity \\
$\mathrm{A}^{ \pm}+\mathrm{B}^{ \pm} \rightarrow \ldots \ldots$ & Effect of electrical charge \\
$\mathrm{A}+\mathrm{B} \rightarrow \ldots \ldots$ & Double energy transfer - cage effect \\
$\leftarrow \mathrm{A}+\mathrm{B} \rightleftarrows \ldots \ldots \ldots$ & Excimers-exciplexes \\
\hline
\end{tabular}

\section{I.1. Smoluchowski Equation Validity Limits}

The Smoluchowski equation assumes that no correlation exists between molecular position and velocity. This hypothesis seems reasonable for large values of time. But for short times a correlation can be significant and leads to kinetic results very different from those obtained usually.

For this purpose, a model has been proposed based on molecular dynamics for the ideal case where each collision is supposed to be effective towards the considered reaction (3). The fluid is considered as a set of hard spheres with a diameter $\sigma$. In the same way, the two reaction partners A and B are supposed to be hard spheres with the same diameter. From all statistically probable configurations, $\Phi$, the probability, $P_{A}(t)$ of existence of a molecule $\mathrm{A}$, is determined in an infinite environment of $\mathrm{B}$.

This calculation is based on the knowledge of distribution evolution of about 500 molecules in space and time. When a molecule leaves the observation cell, it reappears classically on the other side. The simulation uses a constant number of molecules: solvent, A, B in a "laboratory" system.

By deriving $p_{A}(t)$, in the hypothesis of a purely diffusion controlled reaction, the value of the apparent rate of reaction $k_{a}(t)$ is defined by:

$$
\frac{d p_{A}(t)}{\mathrm{d} t}=-k_{a}(t) p_{A}(t) \rho_{B}
$$

where $\rho_{B}$ is the B density in the medium.

Moreover, the Smoluchowski equation in this very simple case, i.e.:

i) reaction between $\mathrm{A}$ and $\mathrm{B}$ at distance $\sigma$

ii) lack of intermolecular potential for $r>\sigma$ 


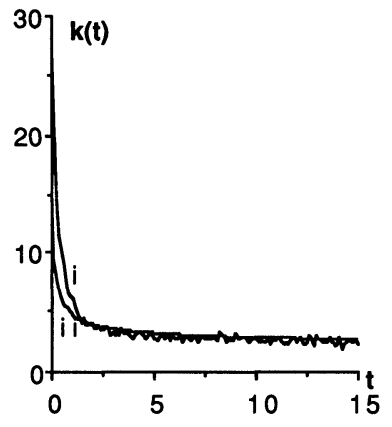

Figure 2 Time dependent rate coefficient, $k_{a}(t)$, in time reduced unit $\left(\left[\mathrm{s} 2 \mathrm{~m} / \mathrm{k}_{\mathrm{B}} \mathrm{T}\right] .5\right.$ where $m$ is the molecular mass). (i) Molecular dynamics result; (ii) Smoluchowski theory.

leads to the expression of $k_{a}(t)$, noted $k_{a}^{s}(t)$ :

$$
k_{a}^{s}(t)=4 \pi N \sigma D\left[1+\frac{\tau}{\sqrt{\pi D t}}\right]
$$

As shown on Figure 2, in a fluid medium as cyclohexane, $k_{a}(t)$ and $k_{a}^{s}(t)$ are very close together for time greater than $10 \mathrm{ps}$.

This important result has several consequences:

i) at first, it points out the interest of the continuous description because of its easy use (numerous softwares exist to solve the differential equations) for time greater than $T_{1}$ corresponding to the end of the decorrelation between velocity and position.

ii) it also shows that, in the case of purely diffusion limited reaction, the measurements in pulsed excitation and in temporal resolution lead to a correct analysis of the kinetics $\left(t>T_{1}\right)$.

iii) the measurements in continuous excitation which take into account the entire life of the emissive molecules $\mathrm{A}$, lead to results which can not be treated with this equation. This statement will be all the more true as the $\mathrm{B}$ concentration is larger.

iv) when the reaction is completely defined by a diffusion limited process, several molecular collisions are necessary for good probability of reaction between A and B.

Under these conditions, the effects illustrated on Figure 2 are still weak, which obviously allows for all temporal domains and then for all measurements in continuous excitation, the use of the Smoluchowski equation. With these considerations, it is possible to use it to treat, at the molecular scale, the coupling between molecular transport and reactivity.

\section{I.2. Notion of Bimolecular Rate of Reaction}

Let us consider the rate of reaction between a pair of molecules $A$ and $B$, located at a distance $r$. Taking account the configuration $\Phi_{0}(r)$, the expression of the chemical rate of a bimolecular process is:

$$
k_{c}=4 \pi N \int_{\sigma}^{\infty} k(r) \Phi_{0}(r) r^{2} \mathrm{~d} r \quad\left(\mathrm{~mole}^{-1} 1 \mathrm{~s}^{-1}\right)
$$


The time evolution of $\Phi$, due to the coupling between transport and reactivity leads to the apparent rate constant of reaction:

$$
k_{a}(t)=4 \pi N \int_{\sigma}^{\infty} k(r) \Phi(r, t) r^{2} \mathrm{~d} r
$$

According to the hypothesis of the model ${ }^{4,5}$ this relation leads to different expressions. The advantage of this formalism is to include the effects of a local potential (real or effective), derived from liquid state physics for example, in an unique expression. However it excludes the physical model of $k(r)$ which is known in some limited cases (long or short range interaction).

The prime problem of this type of system arises when it is possible to choose many parameters which cannot be directly related to the experimental results. Then the singleness of the solution for $k(r)$ fitting the experimental results cannot be proved. It is a domain of inductive approach where some conflicting theories occur.

\section{I.3. General Formalism of a Simple Bimolecular Reaction}

If the reaction

$$
\mathrm{A}+\mathrm{B} \longrightarrow \ldots . .
$$

is considered and if photophysical measurement techniques are used, one obtains:

-before $t=0$ a distribution $\Phi_{0}$ depending or not of local potential

- for $t>0$ a distribution $\Phi(r, t)$ depending on $k(r), D(r)$, intermolecular potential, etc.

It is also possible to start with a nonstationary distribution for $t=0$ as in the case of cage effect phenomena.

In an infinite space with spherical symmetry, the following equation must be solved:

$$
\frac{\partial \phi}{\partial t}=\operatorname{div}\left[D \cdot \operatorname{grad} \Phi-X \frac{D}{k_{B} T} \Phi\right]-k(r) \Phi
$$

with boundary conditions:

$$
\begin{aligned}
& \left(\frac{\partial \phi}{\partial r}\right)_{\sigma}=\left[D \nabla \Phi_{\sigma}+\frac{D}{k_{B} T} \Phi_{\sigma} \nabla X\right]=0 \\
& (\Phi)_{r \rightarrow \infty}=1
\end{aligned}
$$

For a simple bimolecular reaction, a standard expression of the apparent rate constant, at "large" time, such as

$$
k_{a}(t) \approx \alpha\left(1+\frac{\beta}{\sqrt{t}}\right)
$$

is found whatever the assumptions on $D, \Phi_{0}, X, k(r)$, where $\alpha$ and $\beta$ are parameters. Table 2 illustrates, on some examples, the analytical expressions of $\alpha$ and $\beta$. The real problem, as shown later, arises from the experimental results: how it is possible, knowing $\alpha$ and $\beta$, to find values for $D(r), k(r), \mathrm{X}(r) \ldots$ with a physical meaning. 
Table $2 \alpha$ and $\beta$ expressions for different models of photophysical systems

\begin{tabular}{llll}
\hline Conditions & $\alpha$ & $\beta$ & Reference \\
\hline $\begin{array}{l}\text { Contact reaction } \\
\text { Diffusion controlled kinetics, }\end{array}$ & $\begin{array}{l}k_{d}=4 \pi N \sigma D \\
\text { Contact reaction coupling }\end{array}$ & $X=0, g(r)=1, r \geq \sigma$ & $\frac{\sigma}{\sqrt{\pi D}}$ \\
$\begin{array}{l}\text { between transport and } \\
\text { reactivity }\end{array}$ & & \\
$X=0, g(r)=1, r \geqq \sigma$ & $\frac{k_{d} \cdot k_{c}}{k_{d}+k_{c}}$ & $\frac{k_{c}}{k_{d}+k_{c}} \cdot \frac{\sigma}{\sqrt{\pi D}}$ & $(4)$ \\
$\begin{array}{l}\text { Reaction between } \sigma \text { and } \sigma^{\prime} \\
k(r)=k \text { for } \sigma \leq r \leq \sigma^{\prime}(5)\end{array}$ & $\frac{k_{d^{\prime}} \cdot k_{c}}{k_{d^{\prime}}+k_{c}}$ & $\frac{k_{c}}{k_{d^{\prime}}+k_{c}} \cdot \frac{\sigma}{\sqrt{\pi} D}$ & \\
$X=0, g(r)=1, r \geq \sigma$ & & \\
$\begin{array}{l}\text { Contact reaction } \\
\text { Diffusion controlled kinetics }\end{array}$ & $\frac{k_{d}}{\sigma}\left[\int_{\sigma}^{\infty} \frac{\mathrm{d} r}{r^{2} g(r)}\right]^{-1}$ & function of $g(r)$ & (7) \\
$g(r) \neq 1$ & Complex & Complex & \\
$\begin{array}{l}\text { Ionic contact reaction } \\
g(r) \neq 1 \quad X \neq 0\end{array}$ & but calculable & but calculable & (8) \\
General case & Idem & Idem & (9) \\
\hline
\end{tabular}

This problem is usual and it is necessary to introduce external information to reach, in a molecular formalism, a physical description of the reactive system. This dual ambiguity is more emphasized when the space where the reaction occurs is limited. Then, even if a phenomenology explains the analytical expression of $k_{a}(t)$, it is necessary to undertake supplementary studies to limit the number of fitting parameters; it can be the measurement of the radial distribution $g(r)$, obtained from $\mathrm{X}$-ray diffusion experiments, the knowledge of $D$ by different techniques (Taylor method, photobleaching). Outside this experimental approach, a simple model allows, as later shown, the calculation of $X(r)$ which is used for kinetics refinements.

\section{I.4. Presentation of Some Methods for Calculation of X(r)}

Several empirical models describe accurately the case of the interaction of two ground-state molecules. For the interaction potential between a ground-state molecule and an electronically excited molecule, an empirical method is out of the question because of the lack of data and of the specific nature of the excitation of each molecule. Then molecular orbitals theory and perturbation treatment are necessary but some limitations appear especially when the molecules are close together.

\section{I.4.1 SCF ab initio method}

A proper approach of this problem would be the application of modern computational techniques, both perturbation and SCF ab initio (Self consistent field) methods with interaction of configuration. This methodology usually adopted is the so-called "supermolecule" approach in which potential surfaces are calculated for the bimolecular complex and for the isolated molecules. The interaction potential is then the difference between these surfaces. This method has been applied to small systems and the results are compared favorably with experiments if care is taken in 
the selection of the basis sets. The construction of a complete potential surface would be an enormous task and prohibitively expensive for large molecular systems. Fortunately, less rigorous approaches which depend more on empirical input have been applied to the calculations of intermolecular interaction of larger systems.

\section{I.4.2 Molecular dynamics methods for determination of ground state- ground state interaction}

I.4.2.1 Lennard-Jones models In these models, the intermolecular potential is assumed to have a form:

$$
X(r)=\frac{n^{n /(n-m)}}{(n-m)^{m /(n-m)}} * W *\left\{(\sigma / r)^{n}-(\sigma / r)^{m}\right\}
$$

where $W$ is the maximum well depth, $\sigma$ is a radial distance when the potential cancels out, $r$ is the distance between two molecular centers of mass. The two terms in the brackets correspond respectively to the repulsive and dispersive contributions. Usually the value of $m$ is fixed at 6 . Then the dependence of $n$ and $W$ with respect to the orientation of the molecule must be determined.

I.4.2.2 Kihara models ${ }^{10}$ In this model, the molecule is taken as being rigid with a specific shape. The intermolecular potential is a function of the shortest distance between the cores setting the molecule model. The difficulty arises in the relationship between the molecular geometry and the core representation. On the other hand, an explicit dependence of the well depth $W$ on the orientation of the molecules is not known. Thus the Kihara model smooths the orientational effects.

An extension has been proposed by Berne ${ }^{11}$ and called the overlap model. The molecular shape is characterized by an ellipsoidal electron density, described by gaussian distributions and the repulsive energy is taken to be proportional to the overlap of these ellipsoids. The exact forms for $W$ and $\sigma$ as function of the orientation have been given by MacRury. ${ }^{12}$ The lack of experimental data does not provide a precise determination of this dependence like in the Kihara model.

I.4.2.3. Atom pair models ${ }^{13}$ The potential interaction between molecules is considered here as the sum of the interaction potentials of each of the atom in one molecule with all the atoms in the other. The atom-atom interaction is considered as:

$$
U_{i j}=-\frac{A_{i j}}{r_{i j}^{6}}+B_{i j} \exp \left(-C_{i j} r_{i j}\right)
$$

where $r_{i j}$ is the distance of the atoms $i$ and $j$, the coefficients $A_{i j}, B_{i j}$ and $C_{i j}$ depending only on the nature of these atoms. ${ }^{13}$ The total interaction energy can be written as:

$$
U_{\text {inter }}=\sum_{i}^{\text {mol } 1} \sum_{j}^{\text {mol } 2} U_{i j}
$$

The above coefficients are derived from heat of sublimation data and crystal packing distance of a great lot of analogous molecular species. The ability of this model to predict experimental data is well established. ${ }^{14}$ This kind of calculation is easy and 
the dependence of the interaction parameters is determined as function of the orientation and the distance of the two molecules even for large species.

I.4.2.2 Molecular orbitals models ${ }^{15}$ Contrary to the previous models, this treatment calculates the interaction between a ground-state and an electronically excited molecule in addition to the ground-state/ground-state interaction. The most unusual and adequate application concerns the conjugated systems where the interaction consists mainly on the overlapping of $p$ orbitals which can be described in terms of the $\pi$ electrons of separate systems. This approach has been developed by Salem. ${ }^{15,16}$ Firstly intermolecular orbitals are built, their energy depending only on the energies of the original $p$ orbitals and on the interaction energy. The latter is expressed as a function of the overlap $S_{q q}$, of $p$ orbitals of the pair of atoms $q$ and $q^{\prime}$ belonging to each molecule and of the interaction integral $\delta_{q q}$, which is assumed to be proportional to $S_{q q^{\prime}}$.

The ground-state interaction energy is more precisely:

$$
\begin{aligned}
E_{\text {int }}= & -2 \sum_{j}^{\text {occ. }} \sum_{\text {all } j^{\prime}} I_{j j}, S_{j j},-2 \sum_{j^{\prime}}^{\text {occ. }} \sum_{\text {all } j} I_{j j}, S_{j j}, \\
& -2 \sum_{j}^{\text {occ. }} \sum_{k^{\prime}}^{\text {non }}\left[\frac{I_{j k^{\prime}}^{2}}{E_{k^{\prime}}-E_{j}}+\frac{\left(E_{k^{\prime}}-E_{j}\right) S_{j k^{\prime}}^{2}}{4}\right] \\
& -2 \sum_{j^{\prime}}^{\text {occ. }} \sum_{k}^{\text {non. }}\left[\frac{I_{k j^{\prime}}^{2}}{E_{k}-E_{j^{\prime}}}+\frac{\left(E_{k}-E_{j^{\prime}}\right) S_{k j^{\prime}}^{2}}{4}\right]
\end{aligned}
$$

where the indices $j$ and $k$ refer to the molecular orbitals of the first molecule, $j^{\prime}$ and $k^{\prime}$ to the second molecule, $E_{j}, E_{k}, E_{j^{\prime}}$ and $E_{k^{\prime}}$ being the corresponding energies.

Solving the secular determinant of the Hamiltonian in Hückel approximation for each molecule, the molecular orbital $\Psi_{j}$ can be developed as a linear combination of atomic orbitals $\Phi_{q}$ :

Hence

$$
\Psi_{j}=\sum_{q} c_{j q} \Phi_{c}
$$

$$
\begin{gathered}
I_{j j^{\prime}}=\sum_{q} \sum_{q^{\prime}} c_{j q} c_{j^{\prime} q^{\prime}} \delta_{q q^{\prime}} \\
S_{j j^{\prime}}=\sum_{s} \sum_{s^{\prime}} c_{j s} c_{j^{\prime} s^{\prime}} S_{s s}
\end{gathered}
$$

where $\left(q, q^{\prime}\right)$ and $\left(s, s^{\prime}\right)$ are pairs of interacting atoms of the two molecules.

The quantity $\delta_{q q^{\prime}}$, is

$$
\delta_{q q^{\prime}}=k S_{q q^{\prime}} \quad \text { with } \quad k=\beta
$$

$\beta$ being the resonance integral in the isolated molecule. 
The interaction energy $E_{\text {int }}^{*}$ between an excited molecule and a ground-state molecule (noted prime) can be written in the same way:

$$
\begin{aligned}
E_{\mathrm{int}}^{*}=E_{\mathrm{int}}+\Delta E_{\mathrm{int}}^{j-k} \\
\Delta E_{\mathrm{int}}^{j \rightarrow k}=-\left[\left|I_{k k^{\prime}}\right|+\left|I_{j j^{\prime}}\right|\right]+\sum_{\text {all } j^{\prime}}\left(I_{j j^{\prime}} S_{j j^{\prime}}-I_{k j^{\prime}} S_{k j^{\prime}}\right) \\
+\sum_{\substack{\text { all } j^{\prime} \\
(\neq j)}} \mid\left[\frac{I_{j j^{\prime}}^{2}}{E_{j^{\prime}}-E_{j}}+\frac{\left.\left(E_{j^{\prime}}-E_{j}\right) S_{j j^{\prime}}^{2}\right]}{4}\right] \\
\left.+\sum_{\substack{\text { all } j^{\prime} \\
(\neq k)}} \mid \frac{I_{k j^{\prime}}^{2}}{E_{j^{\prime}}-E_{k}}+\frac{\left(E_{j^{\prime}}-E_{k}\right) S_{k j^{\prime}}^{2}}{4}\right]
\end{aligned}
$$

The problem consists in determining the atomic orbital overlaps $S_{q q^{\prime}}$, which depend only on the distance of the two atoms $q$ and $q^{\prime}$ and on the relative orientation of the corresponding $p$ orbitals. The calculation for large molecules is time consuming but it is the simplest model able to calculate this kind of interaction.

The limitation of the method arises from neglecting core-core interaction of atoms like hydrogen which have no $p$ orbital. Another difficulty, connected to the previous one, appears at close distance. If an atom of one molecule is very close to the second molecule, it can be considered as belonging to this molecule and so a "supermolecule" is built without physical existence. This problem is very difficult to solve because there is no theoretical test to check this situation and only a much more sophisticated method like $a b$-initio calculation can prevent this kind of error.

I.4.2.5 Hybrid model To avoid the above difficulty, a hybrid scheme is proposed. Knowing that the "atom pair" model is a well adapted technique to take into account the repulsive energy without much computational effort, this method is used to determine the ground-state/ground-state energy. In a second step, the $\Delta E_{\mathrm{int}}^{j \rightarrow k}$ contribution computed by the overlap orbital method is added to calculate the total energy of excited ground-state interaction, this for each geometrical configuration.

This procedure has been used to build up the interaction energy surface in the cases of two molecules (pyrene and 1,2-benzanthracene) for different conformations. The potential curves are shown on Figures 3 and 4 for particular conformations corresponding to the maximum of interaction.

Each configuration is characterized by a length $(r=$ distance between the centers of mass) and five angles ( $\theta$ and $\kappa$ for locating the center of mass of the non excited molecule in reference axes bound to the excited molecule and three Euler angles for defining the relative orientation of these molecules (Figure 5).

Because of the more preferential orientation of the molecules and in order to avoid a prohibitive size of data for collecting the potential map, an averaged value of the energy is calculated, depending only of $r, \theta$ and $\kappa$ :

$$
E_{\mathrm{int}}^{*}(r, \theta, \kappa)=\frac{\sum_{\text {Euler ang }} E_{\mathrm{int}}^{*} \exp \left(-E_{\mathrm{int}}^{*} / k_{B} T\right)}{\sum_{\text {Euler ang }} \exp \left(-E_{\mathrm{int}}^{*} / k_{B} T\right)}
$$




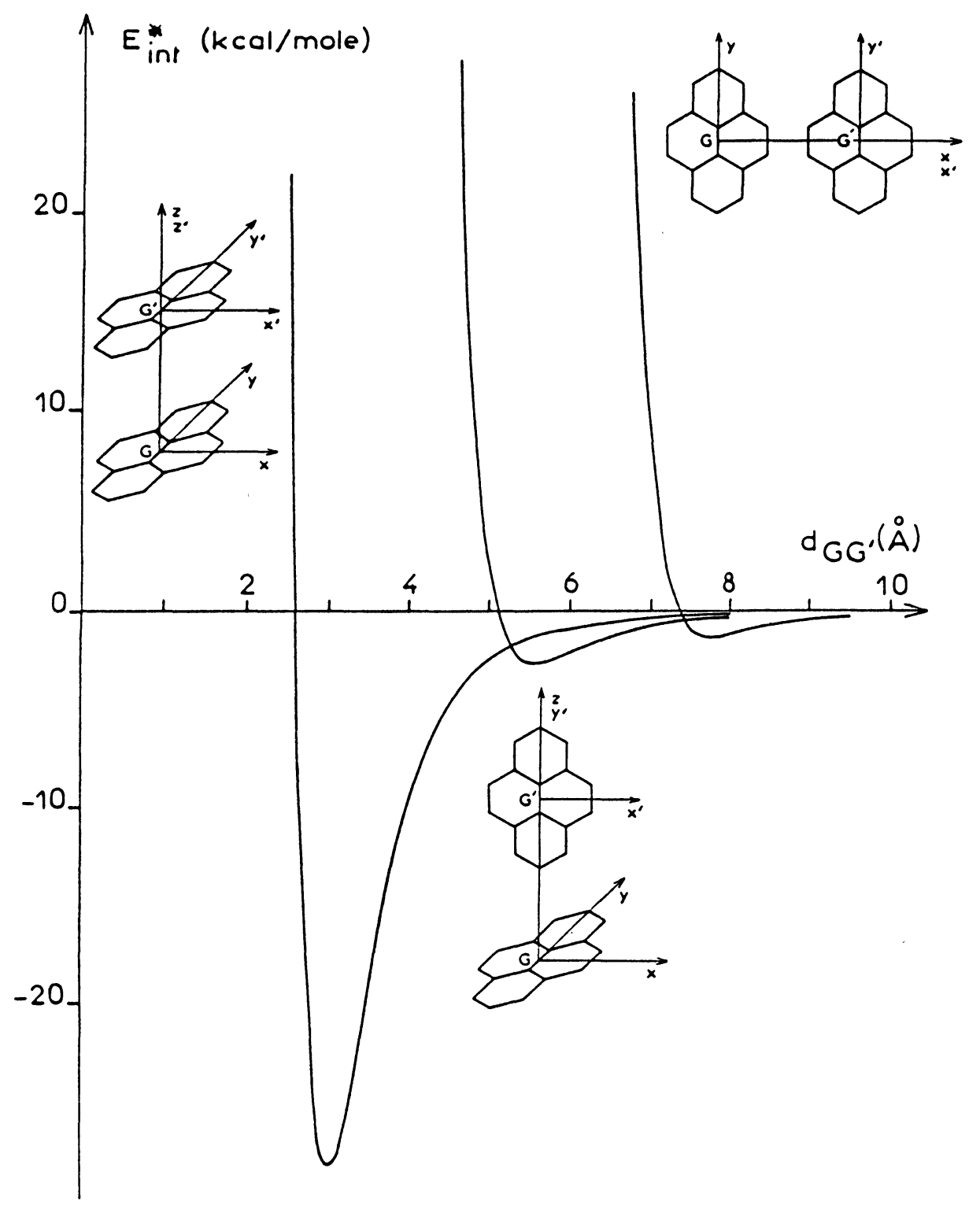

Figure 3 Interaction energy for different configurations of excited pyrene/ground-state pyrene system.

Figure 6 shows the evolution of $E_{\text {int }}{ }^{*}$ for different values of $\theta$ and $\kappa$ in the case of two pyrene molecules.

It must be noted that, in the general case, for a given value of $\theta$, the minimum of potential energy is obtained for a value of $\kappa=0$ which corresponds to a position where the principal axes of the pyrene are parallel. However an exception to this rule is observed on Figure 6 which reveals a relative minimum for $\theta \approx 60^{\circ}$ and $K \approx 20^{\circ}$. 


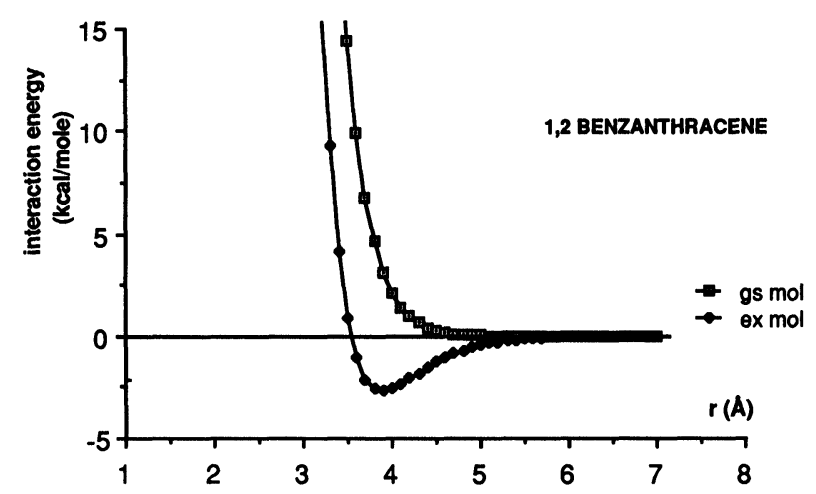

Figure 4 Interaction energy for the more stable configuration of 1,2-benzanthracene for ground-state/ ground-state (gs mol) and excited/ground-state (ex mol) systems.

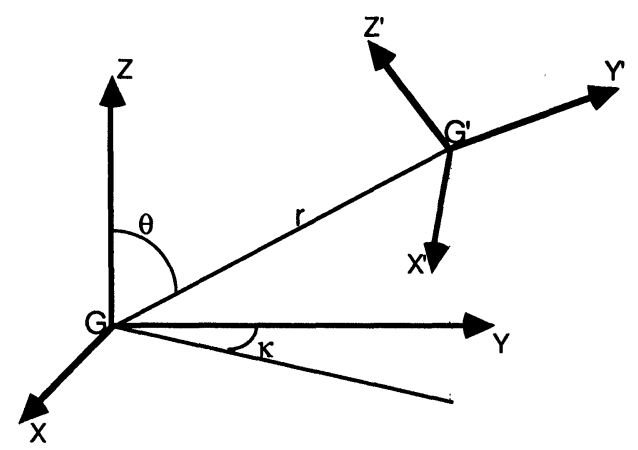

Figure 5 Definition of geometrical parameters used for the calculation of intermolecular potential.

This implies the existence of a second stable excimer different of the known conformer $\left(\theta=0^{\circ}\right.$ and $\left.\kappa=0^{\circ}\right)$. The corresponding energy is however much less important.

In these previous sections, some semi-empirical methods have been presented for the calculation of $X(r)$. Even if the precision is poor, a trend is obtained. Moreover, according to the many parameters which can have an influence on kinetics, important simplifications are necessary as, for example, the choice of an unique space parameter, like the intermolecular distance. Now, if this parameter can allow for the quantitative interpretation of the transport in the scale of large values of $r$, it is not the case for distances close to the molecular radius. Indeed the molecular shapes are not spherical, the interactions are space located.

\section{EXPERIMENTAL METHODS}

As previously mentioned, two approaches are used to study the coupling of transport and reactivity at the molecular scale based on fluorescence emission measurements: 

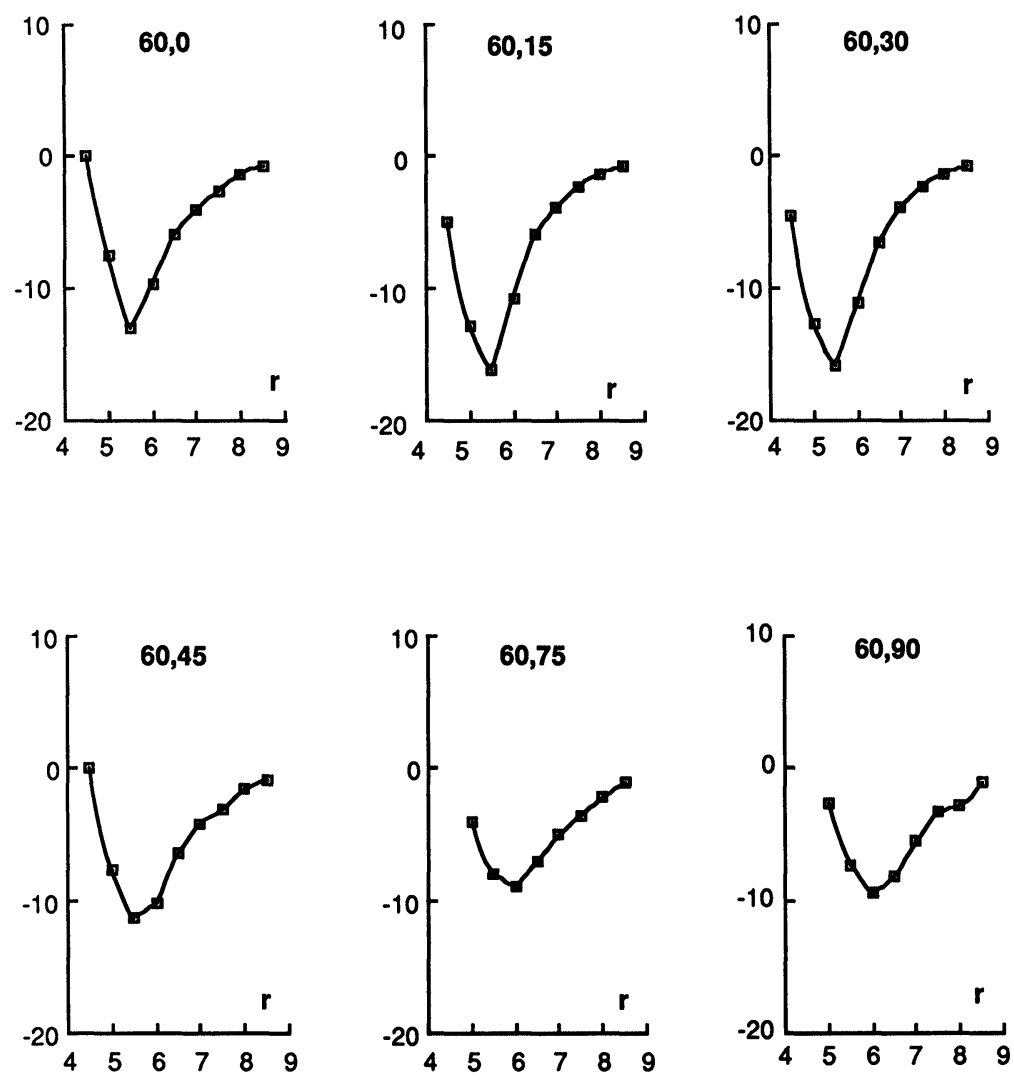

Figure 6 Variations of potential for some relative orientations of pyrene $\left(\theta=60^{\circ}, \kappa=0\right.$ to $90^{\circ}, r$ in $\AA$ ).

-a "continuous" approach, giving a time constant signal, corresponding to the sum of all emitted fluorescent photons,

-a "pulsed" approach, giving the mean time evolution of the fluorescence decay, on scale between 10 picoseconds and a few hundred nanoseconds.

The first one requires a stable light source, with a broad emission spectrum, typically a xenon lamp. The laser role becomes more important in the "pulsed" approach, owing to its property of supplying a pulsed light beam (mode locking or saturated absorption). Two different methods of measuring fluorescence decays are shown hereafter.

\section{II.1. Single Photon Counting}

Figure 7 illustrates a typical assembly: a mode locked ionized argon laser is used as primary source. Then, the pulsed emission $(515 \mathrm{~nm}, 82 \mathrm{MHz})$ pumps a dye laser (here "kiton-red," 620-650 nm, 4-0.4 MHz), including a cavity dumper. The "red" pulse goes through a frequency doubling system to produce a final pulse in the 


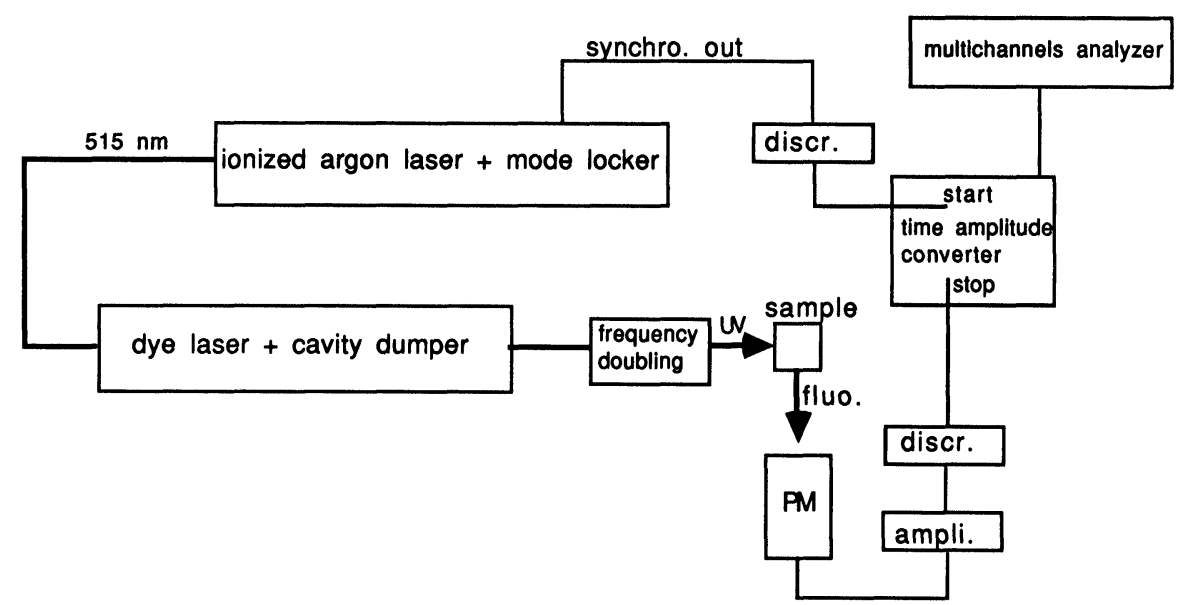

Figure 7 Apparatus assembly used for single photon counting experiments (PM = photomultiplier).

ultraviolet region (where usual interesting molecules absorb), with a half width of less than 10 picoseconds. The single photon counting method consists in accumulating correlation times between a laser pulse and a fluorescent photon, collected on a very sensitive photomultiplier. The treatment of the signal requires high quality electronical devices, inducing nevertheless a response function of the apparatus and, consequently, a good deconvolution program to obtain the real fluorescence decay (as an example, with the above UV pulse, the recorded half width is about 1 nanosecond!). The stability of these devices is still a limiting factor, lifetimes of less than 100-200 picoseconds are not measured accurately.

\section{II.2. Fluorimetry using the Kerr Effect}

The method, illustrated on Figure 8, is totally different. Since it doesn't need sophisticated electronical collecting device, it can be possible, in theory, to reach

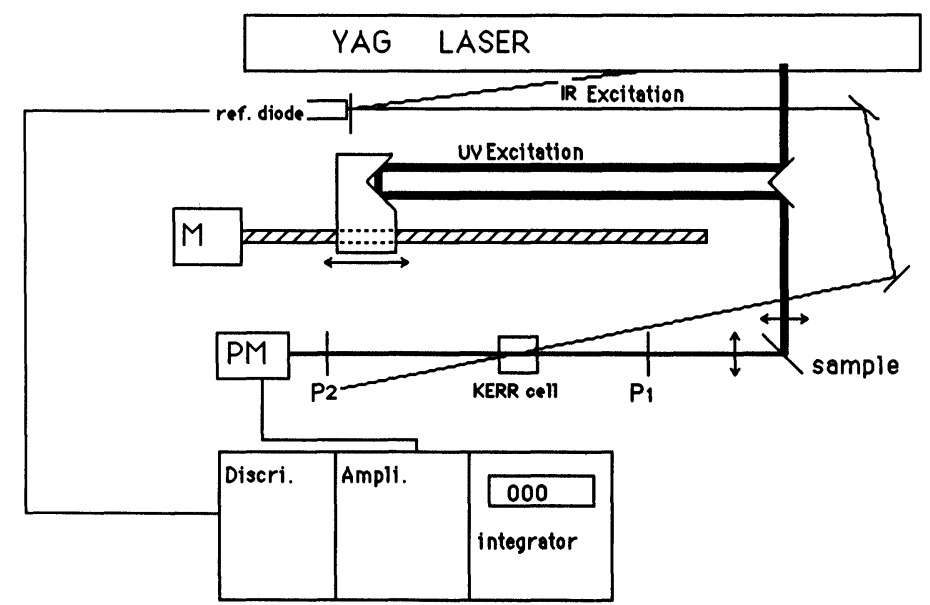

Figure 8 Apparatus assembly for fluorimetry using the Kerr effect $(M=$ step motor). 
lifetimes of a few tens of picoseconds, owing to the less important response function of the system. The Kerr cell contains a liquid $\left(\mathrm{CS}_{2}\right.$ for example) which becomes birefringent when submitted to an intense electric fields. It acts as an "optical shutter" when excited by the infrared pulse of a Yag laser $(1065 \mathrm{~nm})$. A frequency tripling system allows to extract a pulse in the UV region $(355 \mathrm{~nm})$, which excites molecules, the fluorescence of which is collected by a simple photomultiplier on the opposite side of the Kerr cell. A shift between emission and excitation is simply obtained by moving mirrors along the path of the UV pulse. It allows to record the fluorescence decay by a step by step integration. Of course, the low repetition rate of the Yag laser $(10 \mathrm{~Hz})$ is a limiting factor to obtain a precision comparable with the single photon counting method. Moreover, the signal/noise ratio is much smaller and the bad stability of the laser constrains to exclude a quite important number of spurious pulses.

\section{SOME EXPERIMENTAL RESULTS}

From Table 1, some kinetic examples theoretically treated in the first section are illustrated.

\section{III.1. Diffusion Controlled Reactions}

Consider the fluorescence quenching of azulene by biacetyl, studied by continuous and pulsed fluorometry. Steady state measurements were achieved with a Jobin Yvon JY3 spectrofluorometer and fluorescence decay curves were determined with the experimental set up previously described (Section II.1). Decay times obtained after iterative reconvolution ranged from 0.38 to 1.4 nanosecond. Results of the two techniques are presented on Figures 9 and 10. Stern-Volmer plots in steady state show an upward curvature and the quenching increases as the solvent viscosity decreases.

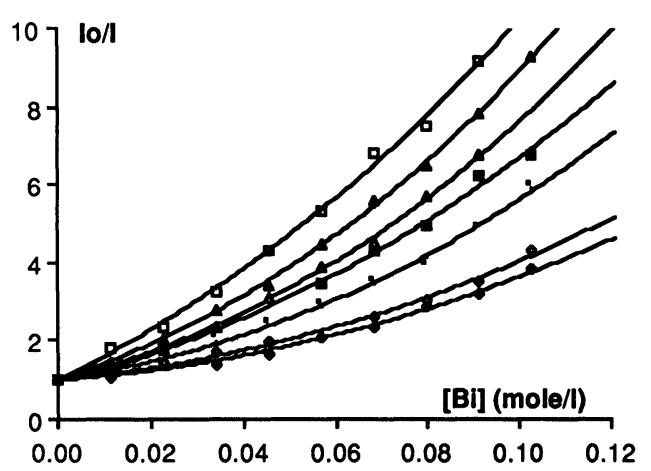

Figure 9 Stern-Volmer plots for quenching of azulene fluorescence by biacteyl at $293 \mathrm{~K}$, in continuous excitation and for different solvants: pentane; $\Delta$ : heptane; + : cyclohexane; $\Delta$ : paraffin/heptane $(30 / 70$ vol); paraffin/heptane (50/50 vol); ०: paraffin/heptane (75/25 vol); •: paraffin/heptane (75/25 vol at $283 \mathrm{~K})$. 


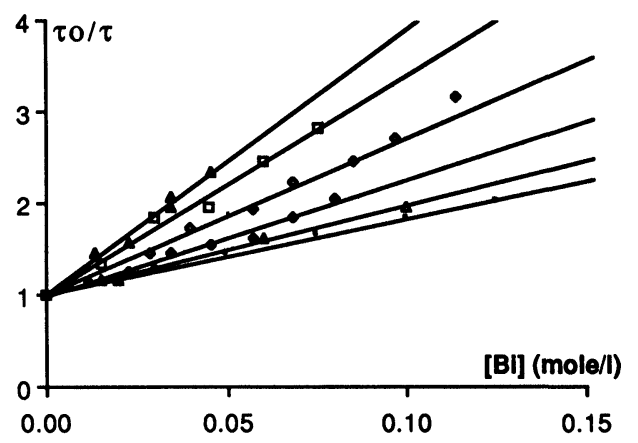

Figure 10 Stern-Volmer plots for quenching of azulene fluorescence by biacteyl at $298 \mathrm{~K}$, in pulsed excitation and for different solvants: heptane; $\bigcirc:$ cyclohexane; $\square$ : paraffin/heptane (55/45 vol); $\Delta$ : paraffin/heptane (70/30); $\Delta$ : paraffin/heptane (75/25 vol); $\Delta$ : pentane at $273 \mathrm{~K}$.

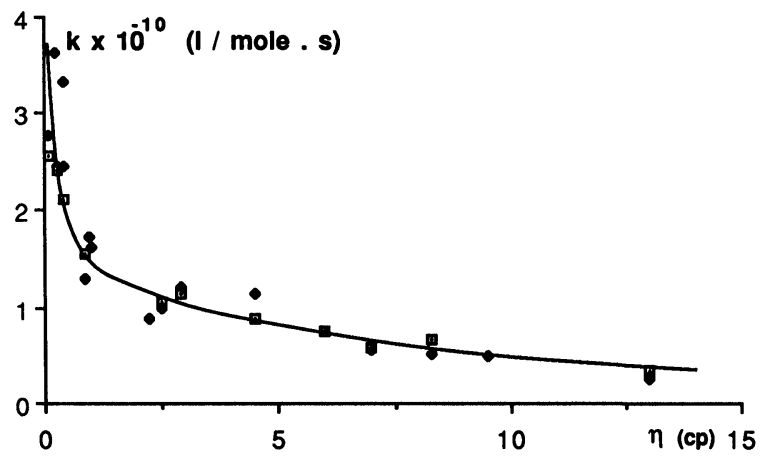

Figure 11 Variations of $k_{\infty}$ versus viscosity of solvant for the system azulene-biacetyl: $\downarrow$ : continuous excitation : $\square$ pulsed excitation.

The same effect of the viscosity on the quenching is observed in transient state experiments. The same effect of the viscosity on the quenching is observed in transient state experiments. Fitting of experimental data points of Figure 9 by a simple model ${ }^{6}$ provide the value of $k_{\infty}$, the rate constant at large times, shown on Figure 11. In the same figure are represented the values of $k_{\infty}$ obtained from the slopes of the curves of Figure 10, showing a good agreement between the two values, in spite of a certain dispersion.

\section{III.2. Coupling between Transport and Reactivity}

Azulene and biacetyl are again supposed to have a space dependent chemical constant, according to a model of Dexter type $\left(k(r)=k_{0} e^{-\mathrm{br}}\right)$. Comparison between this model and a simpler one,${ }^{5}$ which assumes a constant chemical rate between a collision distance $\sigma$ and a reaction distance $\sigma^{\prime}$, allows to compute values of $k_{0}$ and $b$ for each pair. We obtain:

$$
\begin{aligned}
& \text {-azulene-biacetyl: } k_{0}=1.610^{13} \mathrm{~s}^{-1}, b=5 \sigma \\
& \text {-biacetyl-azulene: } k_{0}=1.510^{12} \mathrm{~s}^{-1}, b=3.5 \sigma
\end{aligned}
$$




\section{III.3. Effect of the Electrical Charge}

The fluorescence quenching of fluorescein by $\mathrm{I}^{-}$ions is studied in different wateralcohol mixtures of known dielectric constant $\varepsilon$. Figure 12 shows the evolution of the Stern-Volmer constant $K_{S V}$ with $\varepsilon\left(\mathrm{Io} / \mathrm{I}=1+K_{S V}\left[\mathrm{I}^{-}\right]\right.$, Io being the fluorescence intensity without quencher and I with quencher at the concentration $\left.\left[\mathrm{I}^{-}\right]\right)$.

Quite a good agreement is found between model and experiment when the coupling between transport properties and reactivity is taken into account. In the same way, the difference appearing between results obtained by continuous and pulsed excitation (Figure 13) is partly explained by such a model, including liquid state physics concepts.

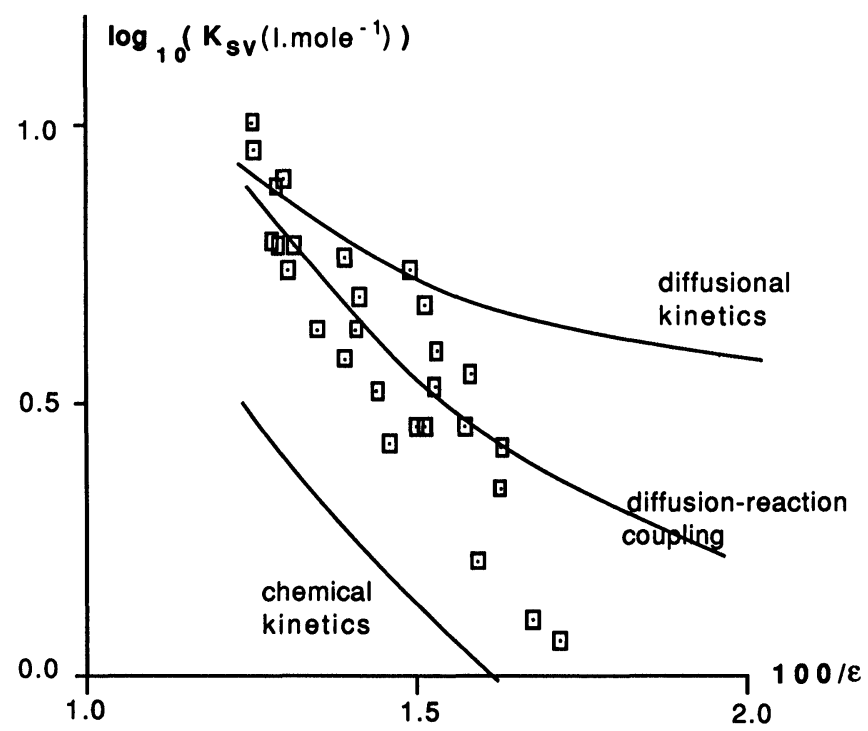

Figure 12 Variations of the slope of the Stern-Volmer plot $\left(K_{S V}\right)$ versus the dielectric constant $\varepsilon$ of the medium (ionic strength $=0.1$ mole/l).

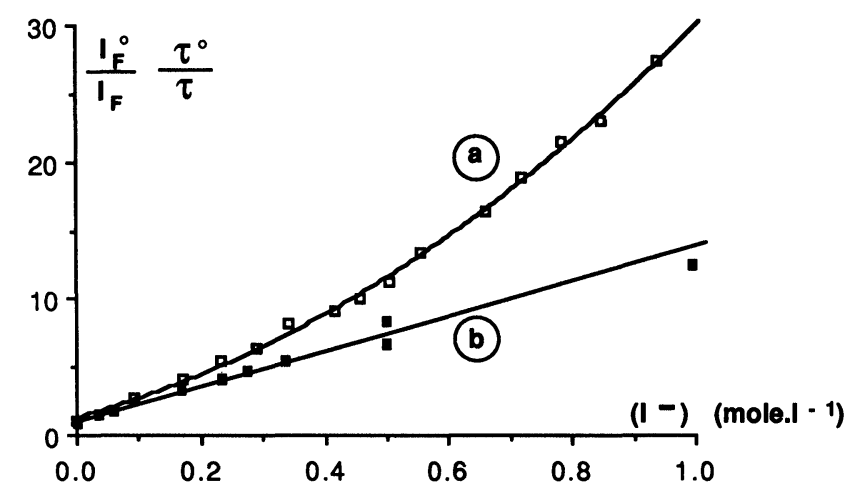

Figure 13 Quenching of the fluorescence emission of fluorescein by iodide ions in water. Confrontation of the experimental data (dots) to the model (full lines) (a) continuous excitation; (b) pulsed excitation. 


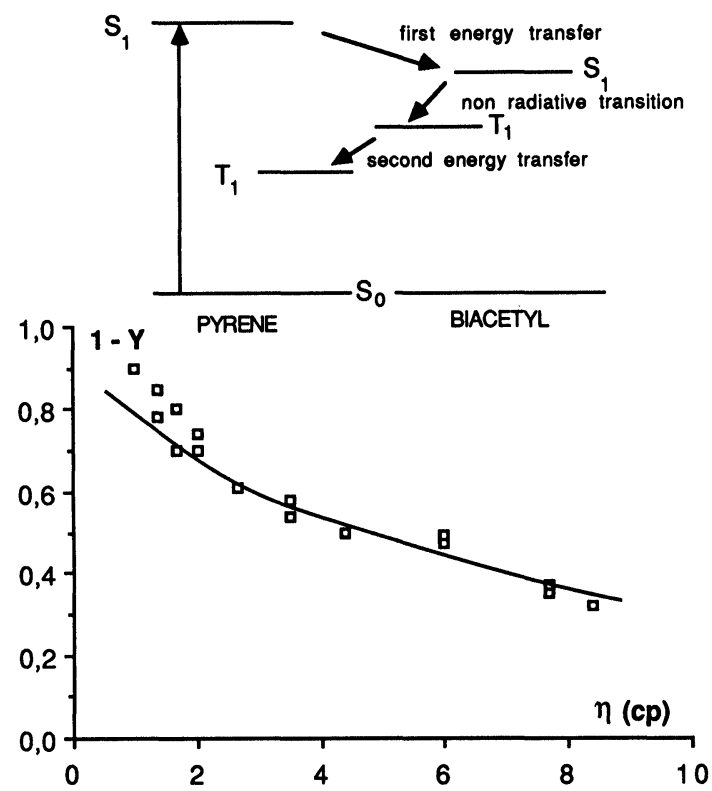

Figure 14 Variations of the second transfer rate (see scheme) versus the viscosity ([biacetyl] $=0.1$ mole/l).

\section{III.4. Double Transfer of Energy}

Two systems involving a double energy transfer have been studied. The second transfer occurs essentially when the two molecules are very near each other, immediately after the first one. The importance of diffusion on the rate of second transfer is clearly shown theoretically as well as experimentally (Figure 14).

\section{III.5. Intermolecular Excimer Formation}

In the case of a reaction such as $\mathrm{A}+\mathrm{B} \longleftrightarrow \mathrm{C}$, when the back reaction becomes important, the apparent rate constant is shown to be no more a decreasing function

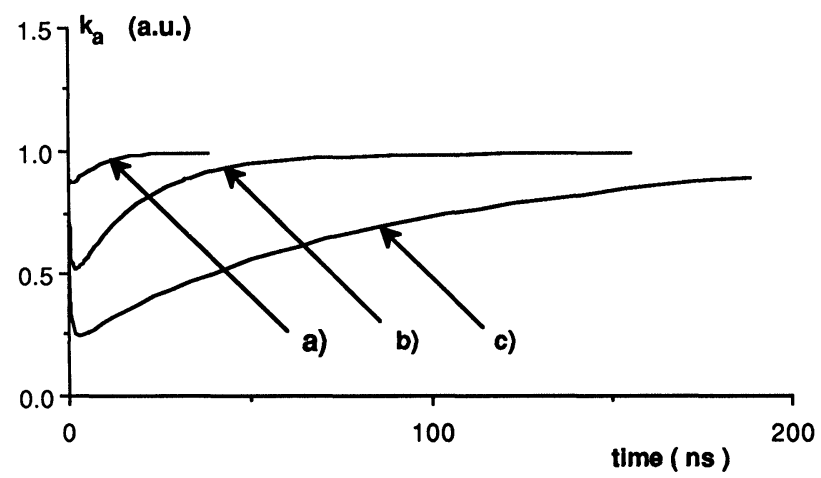

Figure 15 Computed apparent rate constant for a reaction such as $\mathrm{A}+\mathrm{B} \longleftrightarrow \mathrm{C}$ in different solvants: (a) $D=10^{-5} \mathrm{~cm}^{2} / \mathrm{s}$; (b) $D=10^{-6} \mathrm{~cm}^{2} / \mathrm{s}$; (c) $D=10^{-7} \mathrm{~cm}^{2} / \mathrm{s}$. 


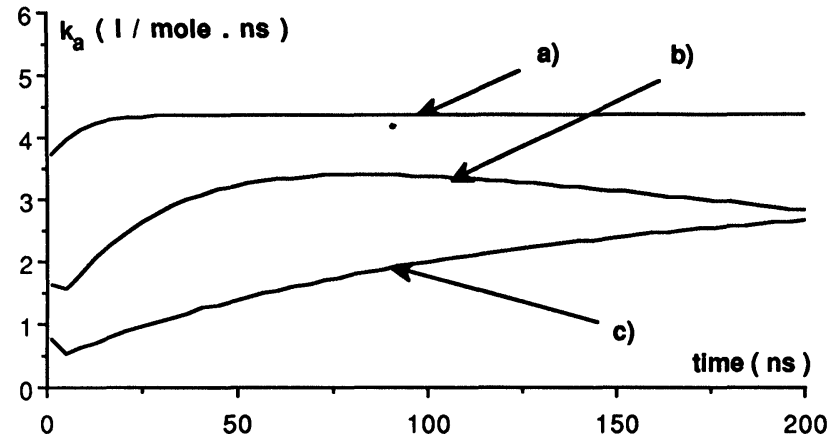

Figure 16 Experimental apparent rate constant for the raction $\mathrm{A}+\mathrm{B} \longleftrightarrow \mathrm{C}$ of 1,2-benzanthracene $(0.02$ mole/l) in different solvants: (a) cyclohexane (viscosity $=0.82 \mathrm{cp}$ ); (b) mixture cyclohexane $(10 \%)$ paraffin $(90 \%)($ viscosity $=9 \mathrm{cp}) ;(\mathrm{c})$ paraffin $($ viscosity $=82 \mathrm{cp})$.

of time, but decreases and then increases, up to a finite value for large times, as indicated on Figure 15.

Experiments have been achieved in pulsed laser excitation on 1,2benzanthracene. An adequate treatment allows to clearly show such a non classical effect (Figure 16).

\section{CONCLUSION}

Photophysical time resolved techniques using lasers allow to observe in a very precise way the coupling of transport and reactivity at the molecular scale. Nevertheless, it is necessary to have a model in order to extract the information. These models are based on the Smoluchowski equation, the use of which implies the knowledge of:

- complex reactive processes,

-local potentials,

- molecular motion,

- cooperative effects,

- order of solvent (when it is not spherical),

-initial distributions, etc.

This is usually not sufficient, as shown in the first part, compared to the information available by experiments. Even if improved experimental systems are still sought, the attention must now be focused on the understanding of reaction mechanisms when molecular transport occurs.

\section{Bibliography}

1. S. A. Rice and A. R. Allnatt, J. Chem. Phys. 34, 2144 (1961).

2. M. V. Smoluchowski, Z. Physik. Chem. 92, 129 (1917).

3. W. Dong, F. Baros and J. C. Andre, J. Chem. Phys. 91, 4643 (1989).

4. F. C. Collins and G. E. Kimball, J. Coll. Sci. 4, 425 (1949).

5. J. C. Andre, M. Bouchy and W. R. Ware, Chem. Phys. 37, 103 (1979).

6. J. C. Andre, M. Niclause and W. R. Ware, Chem. Phys. 28, 371 (1978). 
7. J. C. Andre, F. Baros, M. Bouchy and J. P. Badiali, Chem. Phys. 103, 391 (1986).

8. F. Baros, M. Bouchy, F. Broke and J. C. Andre. Submitted to J. Chem. Soc. Faraday Trans. II.

9. J. C. Andre, F. Baros and A. T. Reis E. Sousa, Int. J. Chem. Kinet, 21, 297 (1989).

10. T. Kihara, Rev. Mod. Phys. 25, 831 (1953).

11. B. J. Berne and P. Pechukas, J. Chem. Phys. 56, 4213 (1972).

12. T. B. Macrury, W. A. Steele and B. J. Berne, J. Chem. Phys. 64, 1288 (1976).

13. R. K. Boyd, C. A. Fyfe and D. A. Wright, J. Phys. Chem. Solids, 35, 135 (1974).

14. F. Mulder and C. Huiszoon, Mol. Phys. 34, 1215 (1977).

15. L. Salem, J. Am. Chem. Soc. 90, 543 (1968).

16. L. Salem, J. Am. Chem. Soc. 90,553 (1968).

\section{APPENDIX}

Bibliography concerning models and experiments on diffusion influenced reactions in tridimensional space (3d) and bidimensional space (2d).

\begin{tabular}{llll}
\hline Reaction & Medium & Models & Experiments \\
\hline $\mathrm{A}^{*}+\mathrm{B}$ & 3d & from [1] to [88] & from (89] to [121] \\
& & & {$[35],[37],[72],[48]$} \\
$\mathrm{A}^{*}+\mathrm{B}$ & 2d micelles & from [122] to [132] & from [133] to [140] \\
& & {$[87],[86],[182]$} & {$[182]$} \\
$\mathrm{A}^{+}+\mathrm{B}^{-}$ & 3d & from [140] to [175] & from [176] to [196] \\
& & {$[10],[13],[16],[28]$} & {$[142]$} \\
Cage effects & 3d & from [197] to [217] & from [218] to [240] \\
& & {$[148],[50]$} & \\
Excimers & 3d, 2d & from [240] to [245] & from [246] to [252] \\
& & {$[87]$} & {$[134]$} \\
\hline
\end{tabular}

1. H. A. Kramers, Physica 7, 284-304 (1940).

2. F. C. Collins, G. E. Kimball, J. Coll. Sci. 4, 425-37 (1949).

3. R. Einsenshitz, Proc. Phys. Soc. 62A (1949).

4. F. C. Collins, J. Coll. Sci. 5, 499-505 (1950).

5. H. L. Frisch and F. C. Collins, J. Chem. Phys. 20, 1797-1803 (1952).

6. H. Fujita, J. Chem. Phys. 21 (1953).

7. R. M. Noyes, J. Chem. Phys. 22, 1349-59 (1954).

8. A. Joblonski, Acta. Phys. Pol. XV, 263-6 (1956).

9. T. R. Waite, Phys. Rev. 107, 463-70 (1957).

10. T. R. Waite, J. Chem. Phys. 28, 103-6 (1958).

11. T. R. Waite, J. Chem. Phys. 32, 21-3 (1960).

12. J. Yguerabide, M. A. Dillon and M. Burton, J. Chem. Phys. 40, 3040-52 (1964).

13. H. L. Friedman, J. Phys. Chem. 70, 3931-3 (1966).

14. J. Yguerabide, J. Chem. Phys. 47, 3049-61 (1967).

15. D. M. Goodall, Ann. Rep. Prog. Chem. 65, 149-63 (1968).

16. J. Z. Steinberg and E. Katchalski, J. Chem. Phys. 48, 2404-10 (1968).

17. J. B. Birks, M. Salete and S. C. P. Leite, Proc. Phys. Soc. London 3, 417-24 (1970).

18. J. R. Cannon, P. C. Du Chateau and D. L. Filmer, Math. Biosciences 9, 61-70, (1970).

19. C. A. Emeis and P. L. Fehder, J. Am. Chem. Soc. 92, 2246-52 (1970).

20. D. Peak, H. L. Frisch and J. W. Corbett, Rad. Effects 11, 149-54 (1971).

21. N. Shigerada, J. Phys. Soc. Japan 30, 233-44 (1971).

22. K. Solc and W. H. Stockmayer, J. Chem. Phys. 54, 2981-8 (1971).

23. F. J. Adrian, J. Chem. Phys. 57, 5107-13 (1972).

24. A. R. Allnatt and L. A. Rowley, Int. Rev. Sci. Phys. Chem. Ser. one. 1, 267-95 (1972).

25. W. G. Burns and A. R. Curtis, J. Phys. Chem. 76, 3008-12 (1972).

26. R. Kaptein, J. Am. Chem. Soc. 94, 6251-62 (1972); J. Am. Chem. Soc. 94, 6262-9 (1972).

27. D. Peak and J. W. Corbett, Phys. Rev. B5, 1226-38 (1972). 
28. J. M. Deutch and B. U. Felderhof, J. Chem. Phys. 59, 1669-71 (1973).

29. C. Lewis and W. R. Ware, Mol. Photochem. 5, 261-85 (1973).

30. K. Solc and W. H. Stockmayer, Int. J. Chem. Kinetics V, 733-52 (1973).

31. G. Wilemski and M. Fixman, J. Chem. Phys. 58, 4009-19 (1973).

32. G. D. J. Phillies, J. Chem. Phys. 60, 976-82 (1974).

33. M. Doi, Chem. Phys. 11, 107-13 (1975).

34. L. Mondrick, J. Chem. Phys. 62, 1907-12 (1975).

35. T. L. Nemzek and W. R. Ware, J. Chem. Phys. 62, 477-89 (1975).

36. M. J. Pilling and S. A. Rice, J. Chem. Soc. Faraday Trans. 2 71, 1563-71 (1975).

37. W. R. Ware, Pure and Applied Chemistry 41 635-59 (1975).

38. B. U. Felderhof and J. M. Deutch, J. Chem. Phys. 64, 4551-63 (1976).

39. Man-Him-Hui and W. R. Ware, J. Am. Chem. Soc. 98, 4712-7 (1976).

40. J. M. Schun and K. S. Schmitz, J. Phys. Chem. 80, 1934-6 (1976).

41. M. Bazin, M. Aubailly and R. Santus, J. Chem. Phys. 67, 5070-3 (1977).

42. B. J. Ackerson, J. Chem. Phys. 69, 684-90 (1978).

43. R. I. Cukier, J. R. Mehaffey and R. Kapral, J. Chem. Phys. 69, 4962-75 (1978).

44. D. L. Emak and J. A. McCammon, J. Chem. Phys. 69, 1352-60 (1978).

45. B. U. Felderhof, J. Phys. A11, 929-37 (1978).

46. S. H. Northrup and J. T. Hynes, J. Chem. Phys. 69, 5246-60 (1978).

47. K. J. Shin and R. Kapral, J. Chem. Phys. 69, 3685-96 (1978).

48. S. M. de B. Costa and A. L. Macanita, J. Photochem. 11, 429-39 (1979).

49. W. P. Helman and K. Funabashi, J. Chem. Phys. 71, 2458-63.

50. S. H. Northrup and J. T. Hynes, J. Chem. Phys. 71, 871-83 (1979); J. Chem. Phys. 71, 884-93 (1979).

51. K. Razi Naqvi, Chem. Phys. Lett. 67, 205-6 (1979.

52. V. Seshadri and V. M. Kenkre, Z. Physik B33, 289-95 (1979).

53. A. I. Burshtein and B. I. Yakobson, Int. J. Chem. Kinetics XII, 201-70 (1980).

54. K. Razi Naqvi, K. J. Mork and S. Waldenstrom, J. Phys. Chem. 84, 1315-9 (1980).

55. J. K. Baird, J. S. McCaskill and N. H. March, J. Chem. Phys. 74, 6812-6 (1981).

56. M. Battezzati and A. Perico, J. Chem. Phys. 75, 886-91 (1981).

57. M. Bixon and R. Zwanzig, J. Chem. Phys. 75, 2354-6 (1981).

58. A. B. Doktorov and N. N. Lukjen, Chem. Phys. Lett. 79, 498-502 (1981).

59. A. Perico and M. Battezzati, J. Chem. Phys. 75, 4430-4 (1981).

60. M. Sitarski, Int. J. Chem. 125-33 (1981).

61. J. Keizer, J. Phys. Chem. 86, 5052-67 (1982).

62. S. Nordholm, J. Chem. Educ. 59, 187-92 (1982).

63. S. H. Northrup, F. Zarrin and J. A. McCammon, J. Phys. Chem. 86, 2314-21 (1982).

64. J. M. G. Martinho and J. C. Conte, J. Luminescence 27, 441-8 (1982).

65. G. Moro and P. L. Nordio, Chem. Phys. Lett. 93, 429-31 (1982).

66. N. G. van Kampen, Int. J. Quantum. Chem., Quantum Chem. Symp. 16, 101-15 (1982).

67. D. F. Calef and J. M. Deutch, Ann. Rev. Phys. Chem. 493-524 (1983).

68. M. Berkowitz, J. D. Morgan, J. A. McCammon and S. H. Northrup, J. Chem. Phys. 79, 5563-5 (1983).

69. B. Goldstein and F. W. Wiegel, Biophys. J. 43, 121-5 (1983).

70. S. Harris, J. Chem. Phys. 78, 4698-700 (1983).

71. R. F. Kayser and J. B. Hubbard, Phys. Rev. Lett. 51, 79-82 (1983).

72. J. Keizer, J. Am. Chem. Soc. 105, 1494-8 (1983).

73. G. Moro and P. L. Nordio, Chem. Phys. Lett. 96, 192-9 (1983).

74. G. P. Morriss and P. T. Cummings, Mol. Phys. 49, 1103-20 (1983).

75. D. Peak, T. C. Werner, R. M. Dennin Jr. and J. K. Baird, J. Chem. Phys. 79, 3328-35 (1983).

76. J. R. Ray, J. Chem. Phys. 79, 5128-30 (1983).

77. G. Sumofer, J. Klafter and A. Blumen, J. Chem. Phys. 79, 5131-5 (1983).

78. S. A. Allison, N. Srinivasan, J. A. McCammon and S. H. Northrup, J. Phys. Chem. 88, 6152-7 (1984).

79. R. D. Astumian and S. A. Schelly, J. Am. Chem. Soc. 106, 304-9 (1984).

80. L. Botar and T. Vidoczy, Chem. Phys. Lett. 104, 16-20 (1984).

81. R. F. Kayser and J. B. Hubbard, J. Chem. Phys. 80, 1127-30 (1984).

82. W. Sung and H. L. Friedman, J. Chem. Phys. 80, 2735-41 (1984).

83. S. I. Temkin and B. I. Yakobson, J. Phys. Chem. 88, 2681-2 (1984).

84. R. D. Astumian and P. B. Chock, J. Phys. Chem. 89, 3477-82 (1985).

85. R. I. Cukier, J. Phys. Chem. 89, 246-52 (1985). 
86. J. Keiser, Acc. Chem. Res. 18, 235-41 (1985).

87. N. L. Thompson and T. P. Burghardt, Biophys. Chem. 21, 173-83 (1985).

88. N. N. Lukzen, A. B. Doktorov and A. I. Burshtein, Chem. Phys. 102, 289-304 (1986).

89. W. R. Ware, J. Phys. Chem. 66, 455-8 (1962).

90. J. T. Dubois and R. L. van Hemert, J. Chem. Phys. 40, 923-5 (1964).

91. W. R. Ware and J. S. Novros, J. Chem. Phys. 70, 3246-53 (1966).

92. P. J. Wagner and I. Kochevar, J. Am. Chem. Soc. 90, 2232-8 (1968).

93. V. A. Blomfield and J. A. Benbasat, Macromolecules 4, 609-13 (1971).

94. N. H. C. Cooke and B. S. Solomon, J. Phys. Chem. 76, 3563-6 (1972).

95. T. Kobayashi and S. Nagakura, Mol. Phys. 23, 1211-21 (1972).

96. B. Nickel, Ber. Buns. Ges. 76, 582-4 (1972).

97. A. J. Robinson, M. A. J. Rodgers, J. P. Keene and C. W. Gilbert, J. Photochem. 1, 379-86(1972/73).

98. W. R. Ware and C. Lewis, J. Chem. Phys. 57, 3546-57 (1972).

99. A. J. Yarwood, Can. J. Chem. 50, 1429-32 (1972).

100. I. B. Berlman, J. Phys. Chem. 77, 562-7 (1973).

101. A. R. Watkins, Chem. Phys. Lett. 29, 526-8 (1974).

102. H. H. Schuh and H. Fischer, Helv. Chim. Acta 61, 2130-64 (1978).

103. J. K. Baird, E. T. Arakawa, D. W. Noid and H. R. Petty, J. Chem. Phys. 71, 5081-9 (1979).

104. N. Selvarajan and V. Ramakrishnan, Indian Journal of Chem. 18A, 340-1 (1979).

105. A. R. Watkins, Chem. Phys. Lett. 65, 380-4 (1979).

106. J. K. Baird and S. P. Escott, J. Chem. Phys. 74, 6993-5 (1981).

107. A. Marty, J. Vigo and P. Viallet, J. Photochem. 16, 203-14 (1981).

108. G. W. Robinson, R. A. Auerbach and J. A. Synowiec, Chem. Phys. Lett. 82, 219-24 (1981).

109. C. C. Wamser, L. Lon, J. Mendoza and E. Olson, J. Am. Chem. Soc. 103, 7228-32 (1981).

110. R. K. Huddleston and W. A. Mulac, J. Phys. Chem. 86, 2279-81 (1982).

111. G. Orlandi, S. Dellonte, F. Flamigni and F. Barigelleti, J. Chem. Soc. Faraday. Trans. 178, 1465-72 (1982).

112. Y. Shimizu and T. Azumi, Studies in Physical and Theoretical Chemistry 27, 137-50 (1982).

113. K. E. Al-Ani and M. Al-Sabti, J. Phys. Chem. 87, 446-9 (1983).

114. P. C. Alford, C. G. Cureton, R. A. Lampert and D. Phillips, Chem. Phys. 76, 103-9 (1983).

115. F. Cartano, A. Lazano, S. Lombrana and E. Martines, Spectrochim. Acta. 39A, 33-5 (1983).

116. T. C. Chan, J. Chem. Phys. 79, 3591-3 (1983).

117. J. M. B. Costa, A. L. Macanita and S. J. Formosinho, J. Phys. Chem. 88, 4089-95 (1984).

118. H. Dreeskamp, A. Laufer and M. Zander, Chem. Phys. Lett. 112, 479-82 (1984).

119. S. Canonica, A. A. Schmid and U. P. Wild, Chem. Phys. Lett. 122, 529-34 (1985).

120. J. Schroeder and J. Troe, Chem. Phys. Lett. 116, 453-9 (1985).

121. J. L. Viovy, J. Phys. Chem. 89, 5465-72 (1985).

122. D. G. Chae, F. H. Ree and T. Ree, J. Chem. Phys. 50, 1581-9 (1969).

123. K. Razi Navqvi, Chem. Phys. Lett. 28, 280-4 (1974).

124. C. S. Owen, J. Chem. Phys. 62, 3204-7 (1975).

125. S. M. B. Costa and A. L. Macanita, J. Phys. Chem. 84, 2408-12 (1980).

126. K. C. Ho and J. R. Maa, J. Colloïd. Interf. Sci. 85, 413-21 (1982).

127. J. Keiser, J. Phys. Chem. 86, 5052-67 (1982).

128. R. D. Astumian and Z. A. Schelly, J. Am. Chem. Soc. 106, 304-9 (1984).

129. R. D. Astumian and P. B. Chock, J. Phys. Chem. 89, 3477-82 (1985).

130. M. D. King and D. Marsh, Biochim. Biophys. Acta. 862, 231-4 (1986).

131. D. A. Pink, D. J. Laidlaw and D. M. Chisholm, Biochim. Biophys. Acta. 863, 9-17 (1986).

132. J. Eisinger, F. Flores and W. P. Petersen, Biophys. J. 49, 987-1001 (1986); A. Arigos, D. F. Calef and J. M. Deutch, Biophys. J. 43, 315-21 (1983).

133. H. J. Galla and E. Sackmann, Biochim. Biophys. Acta. 339, 103-15 (1974).

134. B. M. Liu, H. C. Cheung, K. H. Chen and M. S. Habercom, Biophys. Chem. 12, 341-55 (1980).

135. M. Van Den Zegel, N. Boens and F. C. De Schryver, Biophys. Chem. 20, 333-45 (1984).

136. M. F. Blackwell, K. Gounaris and J. Barber, Biochim. Biophys. Acta. 858, 221-34 (1986).

137. W. L. C. Vaz, R. M. Glegg and D. Hallmann, Biochem. 24, 781-6 (1985).

138. W. L. Vaz, F. Goodsaid-Zalduondo and K. Jacobson, FFBS Letters 174, 199-207 (1984).

139. K. Kano, H. Kawazumi, T. Ogawa and J. Sunamoto, J. Phys. Chem. 85, 2204-9 (1981).

140. R. Fatu, M. Battino, M. D. Esposti, G. P. Castelli and G. Lenaz, Biochem. 25, 3378-90 (1986).

141. P. Debye and E. Huckel, Physik Zeitschrift 24, 305-25 (1923).

142. P. Debye, J. of the Franklin Inst. 224, 135-44 (1937).

143. J. Q. Umberger and V. K. La Mer, J. Am. Chem. Soc. 61, 1099-1109 (1945). 
144. W. H. Stockmayer, J. Chem. Phys. 33, 1291-2 (1960).

145. A. Mozumder, J. Chem. Phys. 48, 1569-5 (1968).

146. A. Mozumder, J. Chem. Phys. 55, 3020-5 (1971).

147. A. Mozumder, J. Chem. Phys. 53, 1326-34 (1971).

148. M. J. Stephen, J. Chem. Phys. 33, 3878-83 (1971).

149. G. C. Abbel and A. Mozumder, J. Chem. Phys. 56, 4079-85 (1972).

150. G. C. Abbel, A. Mozumder and J. L. Magee, J. Chem. Phys. 56, 5422-7 (1972).

151. J. L. Magee and A. B. Tayler, J. Chem. Phys. 56, 3061-6 (1972).

152. D. L. Ermak and Y. Yeh, Chem. Phys. Lett. 24, 243-8 (1974).

153. D. L. Ermak. J. Chem. Phys. 62, 4189-203.

154. G. N. Patey and J. P. Valleau, J. Chem. Phys. 63, 2334-9 (1975).

155. J. S. Hoye and G. Stell, Far. Disc. Chem. Soc. 64, 16-32 (1977).

156. K. M. Hong and J. Noolandi, J. Chem. Phys. 68, 5163-71 (1978).

157. K. M. Hong and J. Noolandi, J. Chem. Phys. 68, 5172-76 (1978).

158. D. Y. C. Chan, D. J. Mitchell and B. W. Niniham, J. Chem. Phys. 70, 2946-57 (1979).

159. P. Colonomos and P. G. Wolynes, J. Chem. Phys. 71, 2644-51; (1979); J. Chem. Phys. 71, 2652-61 (1979).

160. H. L: Friedman, ACS Symp. Series 133, 547-60 (1980).

161. B. Larsen and S. A. Rogde, J. Chem. Phys. 72, 2578-86 (1980).

162. B. R. Sundheim, J. Chem. Phys. 73, 2474 (1980).

163. P. G. Wolynes, Am. Rev. Phys. Chem. 31, 345-76 (1980).

164. M. R. Flannery, Phys. Rev. A25, 3403-6 (1982).

165. K. Holub and A. A. Hornyshev, J. Electroanal. Chem. 142, 57-65 (1982).

166. P. Madden and D. Kivelson, J. Phys. Chem. 86, 4244-56 (1982).

167. I. Ruff, Chem. Phys. Lett. 93, 553-4 (1982).

168. G. N. Patey and S. L. Larnie, J. Chem. Phys. 78, 5183-90 (1983).

169. C. A. Ward, J. Chem. Phys. 79, 5603-15 (1983).

170. L. Belloni, M. Drifford and P. Turq, Chem. Phys. 83, 147-54 (1984).

171. M. Dole, J. Phys. Chem. 88, 6468-9 (1984).

172. P. Clifford, N. J. B. Green and M. J. Pilling, J. Phys. Chem. 88, 4171-6 (1984).

173. M. Mandel and T. Odijk, Ann. Rev. Phys. Chem. 35, 75-108 (1984).

174. R. I. Cukier, J. Chem. Phys. 82, 5457-69 (1985).

175. R. I. Cukier, J. Am. Chem. Soc. 107, 4115-7 (1985).

176. K. Ibuki and M. Nakahara, J. Chem. Phys. 84, 2776 (1986).

177. J. Glowacki and U. Kaminska, Acta Phys. Polonica 23, 43-51 (1963).

178. T. R. Evans, J. Am. Chem. Soc. 93, 2081-2 (1971).

179. G. Corsavo, J. Chem. Educ. 50, 575-6 (1973).

180. P. Doherty and G. B. Benedek, J. Chem. Phys. 61, 5426-34 (1974).

181. A. R. Watkins, J. Phys. Chem. 78, 2555-8 (1974).

182. J. N. Demas and J. W. Addington, J. Am. Chem. Soc. 98, 5800-6 (1976).

183. M. A. J. Rodgers, M. E. Da Silva and E. Wheeler, Chem. Phys. Lett. 43, 587-91 (1976).

184. J. Barthel, R. Wachter and H. J. Gores, Far. Discus. Chem. Soc. 64, 285-303 (1977).

185. R. D. Burkhart, J. Phys. Chem. 81, 370-2 (1977).

186. O. I. Midc and B. Cercek, J. Phys. Chem. 81, 833-7 (1977).

187. J. L. Anderson, F. Raub and A. Morales, J. Phys. Chem. 82, 608-16 (1878).

188. H. P. Haar, U. K. A. Klein and M. Hauser, Chem. Phys. Lett. 58, 525-30 (1978).

189. G. F. W. Seaule and J. Barber, Biochim. Biophys. Acta. 502, 309-20 (1978).

190. H. Shizuka, T. Saito and T. Morita, Chem. Phys. Lett. 56, 519-22 (1978).

191. S. C. Chao, J. Tretzel and F. W. Schneider, J. Am. Chem. Soc. 101, 134-9 (1979).

192. H. Shizuka, M. Nakamura and T. Morita, J. Phys. Chem. 84, 989-94 (1980).

193. C. Biondi and L. Bellugi, Chem. Phys. 62, 145-52 (1981).

194. H. Dreeskamp, A. Laufer and M. Zander, Z. Naturforsch 38a, 698-700 (1983).

195. R. Frank and H. Rau, J. Phys. Chem. 87, 5181-4 (1983).

196. E. M. Kosawer and D. Huppert, Chem. Phys. Lett. 96, 433-5 (1983).

197. N. Periasamy, S. Doraiswamy, G. B. Maiya and B. Venkataraman, J. Chem. Phys. 88, 1638 (1988).

198. T. Koenig, J. Am. Chem. Soc. 91, 2558-62 (1969); G. C. Abbell and A. Mozumder, J. Chem. Phys. 56, 4079-85 (1972).

199. J. F. Garst, J. Am. Chem. Soc. 97, 5062-5 (1975).

200. L. Monchick, J. Chem. Phys. 62, 1907-12 (1975).

201. G. T. Evans and M. Fixman, J. Phys. Chem. 80, 1544-48 (1976). 
202. O. G. Berg, Chem. Phys. 31, 47-57 (1978).

203. A. G. Kofman and A. I. Burshtein, Chem. Phys. 27, 217-28 (1978).

204. J. Noolandi and K. M. Hong, Phys. Rev. Lett. 41, 46-8 (1978).

205. A. I. Burshtein and A. G. Kofman, Chem. Phys. 40, 289-300 (1979).

206. M. Tachiya, J. Chem. Phys. 70, 238-41 (1979).

207. P. J. Wagner, J. Photochem. 10, 387-99 (1979).

208. A. I. Burshtein and B. I. Yakobson, Int. J. Chem. Kinetics XII, 261-70 (1980).

209. J. F. Garst, J. Phys. Chem. 84, 1995-7 (1980).

210. J. T. Hynes, R. Kapral and G. M. Torrie, J. Chem. Phys. 72, 177-88 (1980).

211. V. S. Gurman and V. I. Pergushov, Chem. Phys. 55, 131-5 (1981).

212. A. J. Stace, J. Chem. Soc. Faraday Trans. 2 77, 2105-10 (1981).

213. A. J. Benesi, J. Phys. Chem. 86, 4926-30 (1982).

214. D. J. Dunstein, Phil. Mag. B46, 579-94 (1982).

215. M. W. Balh, C. L. Brook and S. A. Adelman, J. Chem. Phys. 79, 804-15 (1983).

216. C. A. Langhoff, B. Moore and M. De Meuse, J. Chem. Phys. 78, 1191-9 (1983).

217. B. Otto, J. Schroeder and J. Troe, J. Chem. Phys. 81, 202-13 (1984).

218. B. Stevens, J. Phys. Chem. 88, 702-6 (1984).

219. H. Kiefer and T. G. Traylor, J. Am. Chem. Soc. 89, 6667-71 (1967).

220. T. Sawai and W. H. Hamill, J. Chem. Phys. 56, 5524-7 (1972).

221. K. B. Eisenthal, Ber. Buns. Ges. 78, 1105-9 (1974).

222. J. M. Zellweger and H. van den Bergh, J. Chem. Phys. 72, 5405-11 (1980).

223. G. W. Robinson, R. A. Auerbach and J. A. Synowiec, Chim. Phys. Lett. 82, 219-24 (1981).

224. A. J. Stace, J. Chem. Soc. Faraday Trans. 2 77, 2105-10 (1981).

225. D. J. Dunstan, Phil. Mag. B. 46, 579-94 (1982).

226. M. W. Balk, C. L. Brook and S. A. Adelman, J. Chem. Phys. 79 804-15 (1983).

227. P. B. Beeken, E. A. Hauson and G. W. Flynn, J. Chem. Phys. 78, 5892-99 (1983).

228. E. Ghibardi and A. J. Colussi, Chem. Phys. Lett. 94, 121-5 (1983).

229. C. A. Langhoff, B. Moore and M. de Meuse, J. Chem. Phys. 78, 1191-9 (1983).

230. N. J. Turro and G. C. Weed, J. Am. Chem. Soc. 105, 1861-8 (1983).

231. E. B. Aburin, M. V. Encinas, S. Diaz and E. A. Lissi, Int. J. Chem. Kinetics 16, 503-11 (1984).

232. P. Bado, C. Dupuy, D. Magde and K. R. Wilqon, J. Chem. Phys. 80, 5531-8 (1984).

233. I. V. Khudyakov, A. I. Prokofev, L. A. Margulis and V. A. Kuzmin, Chem. Phys. Lett. 104, 409-12 (1984).

234. K. Luther, J. Schroeder, J. Troe and U. Unterberg, J. Phys. Chem. 84, 3072-5 (1984).

235. B. Otto, J. Schroeder and J. Troe, J. Chem. Phys. 81, 202-13 (1984).

236. J. M. Dawes and M. G Sceat, Chem. Phys. 96, 315-26 (1985).

237. H. Hippler, B. Otto, J. Schroeder, V. Schubert and J. Troe, Ber. Buns. Phys. 89, 240-2 (1985).

238. S. Taen, J. Chem. Phys. 85, 1376-81 (1986).

239. T. W. Scott and C. L. Braun, Can. J. Chem. 63, 228-31 (1985).

240. T. W. Scott, Chem. Phys. Lett. 127, 501-4 (1986).

241. S. P. Webb, L. A. Phillips, S. W. Yeh, L. M. Tolbert and J. H. Clark, J. Phys. Chem. 90, 5154-64 (1986).

242. W. D. Weixelbaumer, J. Burbaumer and H. F. Kauffmann, J. Chem. Phys. 83, 1980-2 (1985).

243. S. Lee and M. Karplus, J. Chem. Phys. 86, 1883-903 (1987).

244. J. B. Birks, "Photophysics of aromatic molecules," Wiley-Interscience, New York, 301-71 (1970).

245. K. Sienicki and M. A. Winnik, J. Chem. Phys. 87, 2766-72 (1987).

246. H. Dreeskamp, A. Laufer and M. Zander, Chem. Phys. Lett. 112, 479-82 (1984).

247. J. Richer, G. Lessard, M. Belletete and G. Durocher, Int. J. Chem. Kin. 18, 1163-83 (1986).

248. J. M. Martinho, K. Sienicki, D. Blue and M. A. Winnik, J. Am. Chem. Soc. 110, 7773-7 (1988).

249. P. Collart, S. Toppet and F. C. de Schryver, Macromolecules 20, 1266-71 (1987).

250. J. van den Driessche, P. Palmans, S. Toppet, N. Boens, F. C. de Schryver and H. J. Masuhara,

J. Am. Chem. Soc. 108, 8057 (1984).

251. J. B. Birks, D. J. Dyson and I. H. Munro, Proc. Roy. Soc. A275, 575-88 (1963).

252. J. B. Birks and L. G. Christophorou, Proc. Roy. Soc. A275, 553-64 (1963).

253. J. Roots and B. Nystrom, Eur. Polym. J. 15, 1127-31 (1979).

254. B. Stevens, Adv. Photochem. 8, 161-226, Wiley-Interscience (1971). 